\title{
Chern-Simons Soliton Dynamics in Modular Parameter Space *
}

\author{
Q. Liu \\ Center for Theoretical Physics \\ Laboratory for Nuclear Science \\ and Department of Physics \\ Massachusetts Institute of Technology \\ Cambridge, Massachusetts 02139
}

(May 5, 2018)

Submitted to Physics Letters B

\begin{abstract}
We study dynamics of non-relativistic Chern-Simons solitons, both in the absence and in the presence of external fields. We find that a phase, related to the 1-cocyle of the Galileo group, must be included to give the correct dynamical behavior. We show that interactions among Chern-Simons solitons are mediated by an effective Chern-Simons gauge field induced by the solitons. In the two soliton case, we evaluate analytically the effective interaction Lagrangian, which previously was found numerically.
\end{abstract}

CTP \# 2248

\footnotetext{
*This work is supported in part by funds provided by the U.S. Department of Energy (D.O.E.) under contract \#DE-AC02-76ER03069.
} 
Recently, there has been a lot of study on Chern-Simons solitons [1]. Topological and non-topological solitons are found in a relativistic theory [2,3], and non-topological solitons exist in a non-relativistic model [4]. Since Chern-Simons dynamics is closely related to the quantum Hall effect and perhaps to high- $T_{c}$ superconductivity, it is very interesting to study these solitons [5, 6]. Here, we analyze the dynamics of non-relativistic Chern-Simons solitons in modular parameter space.

Manton originally proposed a method for studying soliton dynamics with specific application to monopole scattering [7]. His idea can be summarized as follows: if there are no forces between static solitons, then at low energies, the dynamics of the full field theory can be described approximately on a finite dimensional space, where degrees of freedom are the modular parameters of the general static solution. This method has been widely applied to many other systems [5,8-11]. In a recent application, the statistical interaction among non-relativistic Chern-Simons solitons is obtained [6].

However, since only first-order time derivatives appear in non-relativistic Chern-Simons theory, it is not clear that Manton's method is directly applicable. Here this question is analyzed and we find that in order to find the correct dynamical behavior of non-relativistic Chern-Simons solitons, a phase, related to the 1- cocycle of the Galileo group, must be introduced when applying the collective coordinate method. Moreover, we use this modified method to study interactions both among well-seperated solitons and between these solitons and external fields.

Let us define the notation: we shall use superscripts $m, n=1, \ldots, N$ as soliton indices, and subscripts $i, j=1,2$ as space indices, for which a summation convention is employed.

We first introduce the Jackiw-Pi Lagragian [1],

$$
L=\int d^{2} \mathbf{r}\left(\frac{\kappa}{4} \epsilon^{\alpha \beta \gamma} A_{\alpha} F_{\beta \gamma}+i \psi^{*} D_{t} \psi-\frac{1}{2}|\mathbf{D} \psi|^{2}+\frac{1}{2 \kappa}\left(\psi^{*} \psi\right)^{2}\right)
$$

where $D_{t}=\partial_{t}+i A_{0}, \mathbf{D}=\nabla-i \mathbf{A}$.

The static solution for $\mathrm{N}$ solitons is

$$
\psi_{s}=\rho^{\frac{1}{2}} e^{i \omega}
$$


with

$$
\rho=\frac{4 \kappa\left|f^{\prime}\right|^{2}}{\left(1+|f|^{2}\right)^{2}}, \quad \omega=\operatorname{Arg}\left(f^{\prime} V^{2}\right)
$$

where

$$
f(z)=\sum_{m=1}^{N} \frac{c^{m}}{z-a^{m}}, \quad V(z)=\prod_{m=1}^{N}\left(z-a^{m}\right)
$$

here $z=x+i y, f^{\prime}=\frac{d f}{d z}$ and $\omega$ is defined in (3) to make the solution nonsingular. Physically, $a^{m}$ is the position of the $m$ th soliton, and $c^{m}$ parametrizes its size and phase. Note, the static solution $\psi_{s}$ satisfies the self-dual equation [1],

$$
\left(D_{1}-i D_{2}\right) \psi_{s}=0
$$

and the action $I=\int d t L$ can also be written as $\mathbb{1 1}$

$$
I=\int d t d^{2} \mathbf{r}\left(i \psi^{*} \partial_{t} \psi-\frac{1}{2}\left|\left(D_{1}-i D_{2}\right) \psi\right|^{2}\right)
$$

Now we discuss the dynamics of these solitons. For sake of simplicity, we hold the $c^{m} s$ time-independent, and let the $a^{m} s$ be time-dependent. First, we notice: because of the Galileo invariance of our model, the static one-soliton solution acquires a phase $\Theta=$ $\mathbf{v} \cdot \mathbf{r}-\frac{1}{2} v^{2} t$ when boosted with a constant velocity $\mathbf{v}$ [1]. Motivated by this fact, we consider the following function,

$$
\psi=\psi_{s} e^{i \Theta}
$$

where $\psi_{s}$ is the self-dual solution (2) and $\Theta$ is a funtion of $\dot{a}^{m}, a^{m}, t$ and $\mathbf{r}$.

We assume that the time-evolution of well-separated Chern-Simons solitons at low energies is approximately described by the effective Lagrangian for the $a^{m} s$, which is obtained by substituting (7) into the original action I (6). Notice that $\psi_{s}$ continues to satisfy equation (5) even with time-dependent parameters, hence we obtain

$$
I_{e f f}=\int d t d^{2} \mathbf{r}\left(-\rho \partial_{t} \Theta-\rho \partial_{t} \omega+\frac{i}{2} \partial_{t} \rho-\frac{1}{2} \rho \partial_{i} \Theta \partial_{i} \Theta\right)
$$


Since $\frac{d}{d t} \int d^{2} \mathbf{r} \rho=0$,

$$
I_{e f f}=\int d t d^{2} \mathbf{r}\left(-\rho \partial_{t} \Theta-\rho \partial_{t} \omega-\frac{1}{2} \rho \partial_{i} \Theta \partial_{i} \Theta\right)
$$

In order to determine $\Theta$, we shall require that near the center of each soliton $\psi$ satisfy the equation of motion of the original Lagrangian to order $\dot{\mathbf{a}}$; we also assume that $\ddot{\mathbf{a}}$ is much smaller than $\dot{\mathbf{a}}$. This leads to,

$$
\Theta=\sum_{m}\left(\dot{\mathbf{a}}^{m}(t) G^{m}(\mathbf{r})\right) \cdot \mathbf{r}
$$

and, $G^{m}(\mathbf{r}) \longrightarrow 1$, when $\mathbf{r}$ is near $\mathbf{a}^{m}$; while $G^{m}(\mathbf{r}) \longrightarrow 0$, when $\mathbf{r}$ is far away from $\mathbf{a}^{m}$. Also the derivatives of $G^{m}$ are order $(\dot{\mathbf{a}})^{2}$, hence can be set to zero. Thus, the effctive action becomes,

$$
I_{e f f}=\int d t d^{2} \mathbf{r}\left(-\sum_{m} \rho \partial_{t}\left(\dot{\mathbf{a}}^{m} \cdot \mathbf{r} G^{m}\right)-\rho \partial_{t} \omega-\frac{1}{2} \sum_{m} \rho\left(\dot{\mathbf{a}}^{m} G^{m}\right) \cdot\left(\dot{\mathbf{a}}^{m} G^{m}\right)\right)
$$

or after an integration by parts,

$$
I_{e f f}=\int d t d^{2} \mathbf{r}\left(\sum_{m} \partial_{t} \rho\left(\dot{\mathbf{a}}^{m} G^{m}\right) \cdot \mathbf{r}-\rho \partial_{t} \omega-\frac{1}{2} \sum_{m} \rho\left(\dot{\mathbf{a}}^{m} G^{m}\right) \cdot\left(\dot{\mathbf{a}}^{m} G^{m}\right)\right)
$$

Here an end point contribution has been dropped. Thus, our effective Lagrangian is,

$$
L_{e f f}=\int d^{2} \mathbf{r}\left(\sum_{m} \partial_{t} \rho\left(\dot{\mathbf{a}}^{m} G^{m}\right) \cdot \mathbf{r}-\rho \partial_{t} \omega-\frac{1}{2} \sum_{m} \rho\left(\dot{\mathbf{a}}^{m} G^{m}\right) \cdot\left(\dot{\mathbf{a}}^{m} G^{m}\right)\right)
$$

We divide $L_{e f f}$ into two parts, $L_{1}$ and $L_{2}$, in which $L_{1}$ is the part induced by the phase $\Theta$ and $L_{2}$ is the part obtained by direct application of Manton's prescription.

$$
\begin{array}{r}
L_{e f f}=L_{1}+L_{2} \\
L_{1}=\sum_{m} \int d^{2} \mathbf{r}\left(\partial_{t} \rho\left(\dot{\mathbf{a}}^{m} G^{m}\right) \cdot \mathbf{r}-\frac{1}{2} \rho\left(\dot{\mathbf{a}}^{m} G^{m}\right) \cdot\left(\dot{\mathbf{a}}^{m} G^{m}\right)\right) \\
L_{2}=-\int d^{2} \mathbf{r} \rho \partial_{t} \omega
\end{array}
$$

We first evaluate $L_{1}$. Using the above described properties of $G^{m}$, we obtain,

$$
\begin{aligned}
L_{1} & =\sum_{m} \dot{\mathbf{a}}^{m} \cdot \int d^{2} \mathbf{r} \partial_{t}\left(\rho G^{m} \mathbf{r}\right)-\frac{1}{2} \dot{\mathbf{a}}^{m} \cdot \dot{\mathbf{a}}^{m} \int d^{2} \mathbf{r} \rho^{m} \\
& =\sum_{m} \dot{\mathbf{a}}^{m} \cdot \frac{d}{d t}\left(\int d^{2} \mathbf{r} \rho^{m} \mathbf{r}\right)-\frac{1}{2} \dot{\mathbf{a}}^{m} \cdot \dot{\mathbf{a}}^{m} \int d^{2} \mathbf{r} \rho^{m}
\end{aligned}
$$


where $\rho^{m}$ is the spherically symmetric one-soliton density for the $m$ th soliton. Finally, using $\int d^{2} \mathbf{r} \rho^{m} \mathbf{r}=4 \pi \kappa \mathbf{a}^{m}$ and $\int d^{2} \mathbf{r} \rho^{m}=4 \pi \kappa$, leaves,

$$
L_{1}=2 \pi \kappa \sum_{m} \dot{\mathbf{a}}^{m} \cdot \dot{\mathbf{a}}^{m}
$$

Not surprisingly, the familiar kinetic energy term for non-relativistic particles is recovered and the mass $4 \pi \kappa$ is exactly what we expect from a consideration of the single-soliton momentum [1].

Now we evaluate $L_{2}$. Notice that $f^{\prime} V^{2}$ can always be written as,

$$
f^{\prime} V^{2}=-\left(\sum_{m=1}^{N} c^{m}\right) \prod_{n=1}^{2 N-2}\left(b^{n}-z\right)
$$

where each $b^{n}$ solves the following equation,

$$
\sum_{m=1}^{N}\left(c^{m} \prod_{n \neq m, n=1}^{N}\left(z-a^{n}\right)^{2}\right)=0
$$

Thus, we have,

$$
\omega=\operatorname{Arg}\left(f^{\prime} V^{2}\right)=\sum_{n=1}^{2 N-2} \operatorname{Arg}\left(b^{n}-z\right)+\mathrm{const}
$$

Using the correspondence between a complex number $z$ and a real 2-dimensional vector $\mathbf{r}$ as well as the formula $\operatorname{Arg}(z)=\theta(\mathbf{r}) \equiv \tan ^{-1}\left(\frac{r_{2}}{r_{1}}\right)$, we have,

$$
\begin{array}{r}
L_{2}=-\sum_{n=1}^{2 N-2} \int d^{2} \mathbf{r} \rho \partial_{t} \theta\left(\mathbf{b}^{n}-\mathbf{r}\right) \\
=-\sum_{n=1}^{2 N-2} \dot{\mathbf{b}}^{n} \cdot \int d^{2} \mathbf{r} \frac{\partial}{\partial \mathbf{b}^{n}} \theta\left(\mathbf{b}^{n}-\mathbf{r}\right) \rho
\end{array}
$$

Recall that in the original theory the Chern-Simons vector potential is given by [1] ,

$$
\mathbf{A}(\mathbf{r}, t)=-\frac{1}{2 \pi \kappa} \int d^{2} \mathbf{r}^{\prime} \nabla \theta\left(\mathbf{r}-\mathbf{r}^{\prime}\right) \rho\left(\mathbf{r}^{\prime}, t\right)
$$

and we see that (20) becomes,

$$
L_{2}=2 \pi \kappa \sum_{n=1}^{2 N-2} \dot{\mathbf{b}}^{n} \cdot \mathbf{A}\left(\mathbf{b}^{n}, t\right)
$$


The interaction among the non-relativistic Chern-Simons solitons is mediated by an effective Chern-Simons vector potential induced by these solitons. A similar result was obtained in the relativistic Chern-Simons model by Kim and Min [5].

We can further simplify (22). As shown in Ref. [1], for the self-dual solution,

$$
\mathbf{A}=-\frac{1}{2} \nabla \times \ln \rho+\nabla \omega
$$

Defining $\Phi(\mathbf{r})=\left(|V|^{2}+|f V|^{2}\right)$, we get,

$$
L_{2}=\left.2 \pi \kappa \sum_{n=1}^{2 N-2} \dot{\mathbf{b}}^{n} \cdot(\nabla \times \ln \Phi(\mathbf{r}))\right|_{\mathbf{r}=\mathbf{b}^{n}}
$$

Combining (16) with (24), we have,

$$
L_{e f f}=2 \pi \kappa \sum_{m} \dot{\mathbf{a}}^{m} \cdot \dot{\mathbf{a}}^{m}+\left.2 \pi \kappa \sum_{n=1}^{2 N-2} \dot{\mathbf{b}}^{n} \cdot(\nabla \times \ln \Phi(\mathbf{r}))\right|_{\mathbf{r}=\mathbf{b}^{n}}
$$

where $\mathbf{b}^{n}$ is determined by solving (18).

In principle, the interaction term $L_{2}$ for a fixed $N$ can be simplified with the aid of (18). As an example, we shall make the simplification for two solitons in their center of mass frame, $\mathbf{a}^{1}=-\mathbf{a}^{2}=\mathbf{a}$, and arbitary constants $c^{1}$ and $c^{2}$. In this case, equation (18) becomes,

$$
\left(c^{1}+c^{2}\right)\left(z^{2}+2 d z a+a^{2}\right)=0
$$

where $d=\frac{c^{1}-c^{2}}{c^{1}+c^{2}}$.

Equation (26) has two roots $b^{1,2}=a\left(-d \pm \sqrt{d^{2}-1}\right)$ while $\dot{b}^{1,2}=\dot{a}\left(-d \pm \sqrt{d^{2}-1}\right)$. Substituting these into the effective Lagrangian and using the explict form of $\Phi$ for two solitons, we obtain, in complex notation,

$$
\begin{array}{r}
L_{2}=-\left.4 \pi \kappa \sum_{n=1}^{2} \operatorname{Im}\left(\dot{b}^{n} \partial_{z} \ln \Phi(z)\right)\right|_{z=b^{n}} \\
=-4 \pi \kappa \sum_{n=1}^{2} \operatorname{Im}\left(\frac{\dot{a}^{n} a^{*}}{|a|^{2}}\left(\frac{(-1)^{n} d\left(\sqrt{d^{2}-1}\right)^{*}}{\left|\sqrt{d^{2}-1}\right|^{2}}+1\right)\right) \\
=-8 \pi \kappa \operatorname{Im}\left(\frac{\dot{a}^{n} a^{*}}{|a|^{2}}\right) \\
=-8 \pi \kappa \frac{d}{d t} \operatorname{Arg}(a)=-8 \pi \kappa \frac{d}{d t} \theta(\mathbf{a})
\end{array}
$$


Recalling that $\theta(\mathbf{a})$ is the relative angle between two solitons, we see that (27) is the statistical interaction with spin $S=-4 \pi \kappa[12]$. This coincides with the result obtained by Hua and Chou with numerical integration [6]. Thus, classically, our Lagrangian describes two freemoving non-relativistic particles with statistical interaction.

As another interesting example, we apply our method to a single Chern-Simons soliton in the presence of external fields. To be specfic, we consider one soliton either in a constant external electric field or in a constant external magnetic field or in a harmonic potential or in any combination of these three. The most general action is

$$
I=\int d t d^{2} \mathbf{r}\left(i \psi^{*} \partial_{t} \psi-A_{0}^{e} \psi^{*} \psi-\frac{1}{2} k r^{2} \psi^{*} \psi-\frac{1}{2}\left|\left(D_{1}^{e}-i D_{2}^{e}\right) \psi\right|^{2}\right)
$$

where $\mathbf{D}^{e}=\nabla-i \mathbf{A}-i \mathbf{A}^{e}, A_{i}^{e}=-\frac{1}{2} \epsilon_{i j} r_{j} B, A_{0}^{e}=-\mathbf{r} \cdot \mathbf{E}$ and $k$ is the strength of the harmonic potential .

In this case, we choose the phase $\Theta$ as follows,

$$
\Theta=\dot{\mathbf{a}} \cdot \mathbf{r}-\frac{1}{8} B^{2} \int^{t} d t^{\prime} \mathbf{a}\left(t^{\prime}\right) \cdot \mathbf{a}\left(t^{\prime}\right)
$$

Then we substitute the trial function (7) with this new $\Theta$ into (28), following similar procedures, we obtain,

$$
L_{e f f}=\frac{1}{2} \dot{\mathbf{a}} \cdot \dot{\mathbf{a}} \int d^{2} \mathbf{r} \rho+\int d^{2} \mathbf{r} \rho \mathbf{E} \cdot \mathbf{r}-\frac{1}{2} k \int d^{2} \mathbf{r} \rho r^{2}+\int d^{2} \mathbf{r} \rho \dot{\mathbf{a}} \cdot \mathbf{A}^{e}(\mathbf{r})
$$

By using the spherical symmetry of the one-soliton solution and the explicit form of $\mathbf{A}^{e}$, we have,

$$
L_{e f f}=2 \pi \kappa \dot{\mathbf{a}} \cdot \dot{\mathbf{a}}+4 \pi \kappa \mathbf{a} \cdot \mathbf{E}-4 \pi \kappa \dot{\mathbf{a}} \cdot \mathbf{A}^{e}(\mathbf{a})-2 \pi \kappa k|\mathbf{a}|^{2}
$$

Here an irrelevant constant term is dropped.

Thus, we see that the Chern-Simons soliton behaves like a non-relativistic point-like particle with charge $4 \pi \kappa$ and mass $4 \pi \kappa$ in these external fields. In fact, exact soliton solutions in the presence of these fields can be found by a coordinate transformation [13]. Our result agrees with the behavior of these solutions. 
In summary, we comment on the phase $\Theta$. From our work, it is clear that this phase plays a very important role in the dynamics of non-relativistic Chern-Simons solitons. However, we have not determined this phase exactly. Thus, it would be very interesting to look for some method to find this phase.

The author thanks Prof. R. Jackiw for suggesting this problem and many helpful discussions. He also thanks D. Bak, Dr. C. Chou and Dr. C. Lee for valuable discussions and comments. 


\section{REFERENCES}

[1] For a review, see R. Jackiw and S.-Y. Pi, Prog. Theor. Phys. Suppl. 107 (1992) 1.

[2] R. Jackiw and E. Weinberg, Phys. Rev. Lett. 64 (1990) 2234;

R. Jackiw, K. Lee and E. Weinberg, Phys. Rev. D42 (1990) 3488.

[3] J. Hong, Y. Kim and P. Y. Pac, Phys. Rev. Lett. 64 (1990) 2230.

[4] R. Jackiw and S.-Y. Pi, Phys. Rev. Lett. 64 (1990) 2969; (C)66 (1991) 2682; Phys. Rev. D42 (1990) 3500.

[5] S.-K. Kim and H. Min, Phys. Lett. B281 (1993) 81.

[6] L. Hua and C. Chou, Phys. Lett. B308 (1993) 286.

[7] N. S. Manton, Phys. Lett. B110 (1982) 54; (C)B154 (1985) 397.

[8] P. J. Ruback, Comm. Math. Phys. 107 (1986) 93.

[9] G. W. Gibbons and P. J. Ruback, Phys. Rev. Lett. 57 (1986) 1492;

R. C. Ferrell and D. M. Eardley, Phys. Rev. Lett. 59 (1987) 1617.

[10] R. Ward, Phys. Lett. B158 (1985) 424;

R. Leese, Nucl. Phys. B344 (1990) 33.

[11] P. J. Ruback, Nucl. Phys. B296 (1988) 669;

T. M. Samols, Phys. Lett. B244 (1990) 285, preprint DAMTP/91-13.

[12] R. MacKenzie and F. Wilczek, Int. J. Mod. Phys. A3 (1988) 2827;

G. Dunne, R. Jackiw and C. Trugenberger, Ann. Phys. (NY) 194 (1990) 197.

[13] For a general review, see S. Takagi, Prog. Theor. Phys. 85 (1991) 463, ibid, 723, (C)86 (1991) 783;

For the applicaton to non-relativistic Chern-Simons theory, see Z. Ezawa, M. Hotta and 
A. Iwazaki, Phys. Rev. Lett. 67 (1991) 441; Phys. Rev. D44 (1991) 452; R. Jackiw and S.-Y. Pi, Phys. Rev. Lett. 67 (1991) 415; Phys. Rev. D44 (1991) 2524 and Ref. [4]. 